\title{
Alterations of the circular RNA profile in the jejunum of neonatal calves in response to colostrum and milk feeding
}

\author{
X. W. Zhao, D. W. Huang, H. L. Zhu, X. C. Pan, X. X. Wang, Y. X. Qi, G. L. Cheng, H. L. Zhao, and Y. X. Yang* \\ Institute of Animal Science and Veterinary Medicine, Anhui Academy of Agricultural Sciences, Hefei, 230031, China
}

\section{ABSTRACT}

Circular RNA (circRNA) have been suggested to contribute to regulating gene expression in various tissues and cells of eukaryotes. However, little is known regarding the expression pattern of circRNA and their potential function in the small intestine of neonatal calves that receive colostrum. In the current study, jejunum tissue samples were collected from control calves ( $2 \mathrm{~h}$ after birth; $\mathrm{CT} ; \mathrm{n}=3$ ) and neonatal calves that ingested colostrum ( $24 \mathrm{~h}$ after birth; $\mathrm{CO} ; \mathrm{n}=3$ ) or milk (24 $\mathrm{h}$ after birth; MK; $\mathrm{n}=3$ ) to compare the circRNA expression patterns using a high-throughput RNA sequencing approach. A total of 21,213, 17,861, and 21,737 circRNA were identified in the $\mathrm{CT}, \mathrm{CO}$, and MK groups, respectively. Only 13,254 of these circRNA were common to the 3 groups, suggesting high specificity of circRNA expression depending on nutrient type. In total, 243, 249, and 283 circRNA were differentially expressed in the $\mathrm{CO}$ versus $\mathrm{CT}, \mathrm{CO}$ versus $\mathrm{MK}$, and MK versus CT comparisons, respectively. Gene ontology analysis showed that the differentially expressed circRNA and their predicted or known target genes from the $\mathrm{CO}$ and $\mathrm{MK}$ groups were mainly involved in macromolecule metabolic process, response to stress, and vesicle-mediated transport. Moreover, pathway analysis showed that the Rap1 signaling pathway, focal adhesion, ubiquitin-mediated proteolysis, and extracellular matrix-receptor interaction were the most significantly enriched pathways. These data collectively indicate that circRNA are abundant and dynamically expressed when calves receive colostrum and act as microRNA sponges to regulate their target genes for jejunum function during the early development of newborn calves.

Key words: circular RNA, colostrum, jejunum, newborn calf

Received November 2, 2018.

Accepted April 10, 2019.

*Corresponding author: yyongxin@yahoo.com

\section{INTRODUCTION}

Colostrum is known as liquid gold for neonatal ruminants because it contains abundant protein, fat, vitamins, minerals, and nucleotides and provides a rich supply of bioactives, especially Ig (Jain et al., 2007; Georgiev, 2008). Maternal Ig cannot readily pass the placental barrier and are therefore barely transferred to the fetus during pregnancy (Godden, 2008); thus, colostrum feeding in the early days of life plays a central role in the passive immune transfer that is crucial for the health and survival of neonatal calves (Kertz et al., 2017). Consequently, many previous studies investigated the effects of the volumes and time of colostrum feeding and the concentrations of colostral Ig on the transfer of passive immunity in neonatal calves (Conneely et al., 2014; Osaka et al., 2014). The findings showed that colostrum not only provides abundant Ig to meet the requirements of passive immunity for neonatal calves but also supplies other substrates to promote intestinal epithelial cell growth and development (Blum and Hammon, 2000; Yang et al., 2015). Moreover, studies of mRNA and microRNA (miRNA) expression in the intestine of mammals indicated that several genes are related to colostrum digestion and absorption, contributing to intestinal development (Sharbati et al., 2010; Liang et al., 2014). Taken together, these studies suggest that the expression of several genes in the neonatal intestine may contribute to the metabolism and absorption of colostral components, especially Ig and other bioactive substrates. However, the effects of colostrum intake on the expression of genes in the small intestine of calves have not been well characterized.

Noncoding RNA such as miRNA and long noncoding RNA are implicated in multiple biological functions (Bartel, 2009; Guttman and Rinn, 2012; Batista and Chang, 2013). Circular RNA (circRNA) have emerged as a novel type of noncoding RNA that develops covalently closed loop structures without $5^{\prime}-3^{\prime}$ polarities (Qu et al., 2015). Circular RNA mainly consist of circular exonic RNA, circular intronic RNA, and intron and exon-intron circRNA, generated by back-splicing or lariat introns (Zhang et al., 2013; Jeck and Sharpless, 
2014). Importantly, circRNA are implicated in the regulation of gene expression at multiple levels. For example, circRNA may function as miRNA sponges or potent competing endogenous RNA molecules and contribute to competitive binding with other competing endogenous RNA (mRNA, long noncoding RNA, or pseudogenes) for regulating target gene expression (Memczak et al., 2013; Taulli et al., 2013; Kulcheski et al., 2016). In particular, a recent study of the cow mammary gland indicated that circRNA derived from CSN1S1 were highly abundant, and casein circRNA contained many miR-2284 family binding sites, which possibly act as miR-2284 sponges that can regulate casein translation (Zhang et al., 2016). However, little information is available concerning the expression of circRNA in the intestinal response to colostrum intake.

The jejunum segment is part of the digestive tract that plays an important role in absorption of colostral components, especially Ig, in neonatal ruminants (Jochims et al., 1994; Moretti et al., 2013). Considering that miRNA could play a key role in regulating the development of the gastrointestinal tract during early life in animals (Sharbati et al., 2010; Liang et al., 2014), we hypothesized that circRNA might also play a role in mediating the jejunum response to colostrum digestion and absorption in calves. Thus, the objective of this study was to investigate the differentially expressed circRNA in the jejunum between calves fed colostrum and milk via high-throughput RNA sequencing analysis. These results may provide novel insight into the circRNA profiles in the gastrointestinal tract related to colostrum feeding, which could facilitate further investigations of the functions of circRNA in the small intestine during the early life of newborn calves.

\section{MATERIALS AND METHODS}

\section{Animals and Sample Collection}

Animal care and use procedures were approved by the Animal Care Advisory Committee of the Anhui Academy of Agricultural Sciences. Male calves from multiparous Holstein cows at Chuzhou dairy farm (Chuzhou, China) were selected for this study, and the detailed experimental procedures were characterized in our previous study (Qi et al., 2018; Zhao et al., 2018). In brief, the animals were divided into 3 groups: the control group (CT) comprising 3 calves that were not fed colostrum or milk, the colostrum group (CO) comprising 3 calves that received 2 colostrum meals at 1 to $2 \mathrm{~h}$ and 10 to $12 \mathrm{~h}$ after birth, and the milk group (MK) comprising 3 calves that received 2 milk meals at 1 to $2 \mathrm{~h}$ and 10 to $12 \mathrm{~h}$ after birth. Colostrum or milk were incubated in a water bath at $40^{\circ} \mathrm{C}$ and fed at approximately $8.0 \%$ of BW. The jejunum samples were collected from the same mid-jejunum segment at approximately $2 \mathrm{~h}$ after birth for control calves and 24 $\mathrm{h}$ after birth for the CO and MK groups. Samples were washed with precooled PBS $\left(4^{\circ} \mathrm{C}\right)$, snap-frozen in liquid nitrogen, and then stored at $-80^{\circ} \mathrm{C}$ until analysis.

\section{Library Preparation for High-Throughput RNA Sequencing}

The tissue sample was manually homogenized into a fine powder with a mortar and pestle in liquid nitrogen, and total RNA was extracted with Trizol reagent (Invitrogen, Carlsbad, CA) according to the manufacturer's instructions. The RNA concentration and quality were evaluated using a NanoDrop 2000 spectrophotometer (Thermo Fisher Scientific, Wilmington, DE) and Agilent bioanalyzer 2010 (Agilent, Palo Alto, CA). Subsequently, total RNA samples (5 $\mu \mathrm{g}$, RNA integrity number $\geq 8.0$ and $28 \mathrm{~S} / 18 \mathrm{~S} \geq 1.0$ ) were treated with the Ribo-Zero magnetic kit (Epicenter, Madison, WI) to remove rRNA and then incubated at $37^{\circ} \mathrm{C}$ for $1 \mathrm{~h}$ in a $100-\mu \mathrm{L}$ reaction with $20 \mathrm{U}$ of RNase $\mathrm{R}$ (Epicenter). The remaining RNA was used for library preparation using the NEB NextUltra Directional RNA Library Prep Kit for Illumina (New England Biolabs, Ipswich, MA). Circular RNA were randomly fragmented using a fragmentation buffer, and the first-strand cDNA was synthesized using random hexamer primers and reverse transcriptase. Second-strand cDNA synthesis was subsequently performed using DNA polymerase I and RNase H. Remaining overhangs were converted into blunt ends via exonuclease and polymerase activities. After adenylation of the 3 '-ends of DNA fragments, NEBNext Adaptor (New England Biolabs) with a hairpin loop structure was ligated to prepare for hybridization. To select appropriate insert fragments of preferentially 150 to $250 \mathrm{bp}$ in length, the library fragments were purified with AMPure XP Beads (Beckman Coulter, Beverly, MA) and then incubated with $3 \mu \mathrm{L}$ of User Enzyme (New England Biolabs) at $37^{\circ} \mathrm{C}$ for 15 min before PCR, which was performed with Phusion High-Fidelity DNA polymerase, universal PCR primers, and index (X) primer. Finally, the PCR products were purified (AMPure XP system) and library quality was assessed using an Agilent Bioanalyzer 2100 and quantitative PCR. The qualified library was sequenced on the Hiseq2500 platform (Illumina, San Diego, CA).

\section{CircRNA Identification}

Raw sequencing data in FASTQ format were first processed through in-house Perl scripts, and clean data were obtained by removing reads containing adapters or 
Table 1. Primers for reverse-transcription quantitative PCR

\begin{tabular}{|c|c|c|}
\hline Gene name ${ }^{1}$ & Sequence & $\begin{array}{l}\text { Product } \\
\text { length (bp) }\end{array}$ \\
\hline$G O P C-\mathrm{F}$ & CTATGTTCCGGTGGCTGGAG & 172 \\
\hline$G O P C-\mathrm{R}$ & GTCTGGGCTTTGTGGCAAAG & \\
\hline$S M G 6-\mathrm{F}$ & AATTGGAGCTGAGCCCTGAC & 239 \\
\hline$S M G 6-\mathrm{R}$ & CCGGGTAAACAGCTTCCCAT & \\
\hline$H I P K 1-\mathrm{F}$ & TGCCCTTTACTCTGAGCTGC & 234 \\
\hline$H I P K 1-\mathrm{R}$ & ACTGGTTGCCATGAGAGTGG & \\
\hline HEPACAM2-F & AGTTCACCGACGAAGGCAAT & 157 \\
\hline HEPACAM2-R & TTTCCCACGTACTCCACAGC & \\
\hline$H L C S-\mathrm{F}$ & GAGGGTCAATGTCATGGGCA & 199 \\
\hline$H L C S-\mathrm{R}$ & GATGACCAGCAGCAGACAGT & \\
\hline$G A P D H-\mathrm{F}$ & AAGTTCAACGGCACAGTCAA & 119 \\
\hline$G A P D H-\mathrm{R}$ & TACTCAGCACCAGCATCACC & \\
\hline
\end{tabular}

poly-N (>5\%) and poor-quality reads (quality score < 20). All downstream analyses were based on the cleaned data with high quality confirmed. Filtered data were mapped to the Bos taurus reference genome sequence (ensembl.org/Bos_taurus/) using TopHat2 software, which is an efficient sequence alignment method based on Bowtie software, and the splicing junctions between exons were then identified. Transcript assembly and abundance estimation were performed using Cufflinks (Trapnell et al., 2010), and RNA sequencing read coverage was visualized for selected genes in Integrative Genomics Viewer (Robinson et al., 2011).

Circular RNA Identifier (CIRI) tools were used to predict circRNA. The SAM files were scanned twice, and sufficient information was collected to identify and characterize circRNA. The abundance of circRNA was determined by the number of junction reads identified with CIRI tools. miRanda (version 3.3a; http://www .microrna.org/microrna/home.do) and TargetScan (version 7.0, March 2018 release; http://www.targetscan $. \mathrm{org} /$ ) tools were used to predict the target miRNA of identified circRNA.

\section{Validation of CircRNA Expression Using Reverse- Transcription Quantitative PCR}

The expression levels of selected circRNA obtained from high-throughput RNA sequencing were validated by reverse-transcription quantitative PCR, and GAPDH was used as a reference gene (Ontsouka et al., 2004). Two sets of primers for each circRNA were designed using Primer Express software version 5.0 (Applied Biosystems, Foster City, CA), and details of primers used are summarized in Table 1. Total RNA was extracted using the Super UNIQ-10 Total RNA extraction kit (Sangon Biotech Co. Ltd., Shanghai, China), and cDNA was synthesized using an M-MLV reverse transcription kit (Takara Biochemicals, Dalian, China).
Selected circRNA expression studies using SYBR green quantitative PCR were performed in a LightCycler 480 Multiwell Plate 96 system (Roche Diagnostics, Basel, Switzerland) with the following program: $94^{\circ} \mathrm{C}$ for 30 $\mathrm{s} ; 40$ cycles of $94^{\circ} \mathrm{C}$ for $5 \mathrm{~s}$ and $61^{\circ} \mathrm{C}$ for $35 \mathrm{~s}$; melting temperature $97^{\circ} \mathrm{C}$ for $1 \mathrm{~min}, 60^{\circ} \mathrm{C}$ for $30 \mathrm{~s}$, and $97^{\circ} \mathrm{C}$ for $30 \mathrm{~s}$. The expression level of circRNA was defined based on the threshold cycle $(\mathrm{Ct})$ value, and the relative expression levels were calculated via the $2^{-\Delta \Delta \mathrm{Ct}}$ method.

\section{Data Analysis}

Differential expression analysis of circRNA between treatment groups was performed with Perseus software (http://www.perseus-framework.org) using 2-sample tests. Circular RNA with a $q$-value $<0.05$ and $\log 2$ fold change $\geq 3$ were determined to be significantly differentially expressed.

The DAVID tool (david.ncifcrf.gov) was used to analyze the potential functions of the target genes of the differentially expressed circRNA. The top enriched Gene Ontology (GO) terms for these genes were ranked by enrichment score, and Kyoto Encyclopedia of Genes and Genomes pathway analysis was performed to determine the involvement of linear transcripts in different biological pathways.

\section{RESULTS}

\section{Identification of CircRNA in the Jejunum}

After removing the adapter primer sequences and sequences of low quality, a total of $136.97 \mathrm{~GB}$ of clean reads was obtained from the jejunum tissues, with an average percentage of bases over $88.0 \%$. All of the samples analyzed qualified for subsequent analysis (Supplemental Table S1; https://doi.org/10.3168/jds .2018-15942). 
We detected 36,829 circRNA from the jejunum tissues based on the results of CIRI software, including 21,213 in the CT group, 17,861 in the CO group, and 21,737 in the MK group, with 13,254 circRNA common to the 3 groups. Among the identified circRNA, 67.1, 20.9 , and $12.0 \%$ were generated from exons of a single protein-coding gene, intergenic regions, and introns, respectively. The size of the candidate circRNA ranged from 200 to 20,000 nucleotides, and the identified circRNA in the jejunum tissue were generated from all chromosomes (Supplemental Figure S1; https://doi .org/10.3168/jds.2018-15942).

\section{Identification of Differentially Expressed CircRNA}

The profile plot of the identified circRNA is presented in Supplemental Figure S2 (https://doi.org/10.3168/ jds.2018-15942), demonstrating clear differences in the expression of circRNA of the jejunum tissue among the groups. For better visualization, volcano plots were constructed to represent the distribution of differentially expressed circRNA among the $\mathrm{CO}$ versus $\mathrm{CT}, \mathrm{CO}$ versus MK, and MK versus CT comparisons (Figure 1). After filtering circRNA with a $q$-value $<0.05$ and $\log 2$ fold change $\geq 3,86$ circRNA were found to be upregulated and 157 circRNA were downregulated in the CO group compared with the CT group (Supplemental Table S2; https://doi.org/10.3168/jds.2018-15942). Among these, circ_017958 (AHI1), circ_000781 (HIP1), circ_011322 (HIPK1), circ_033679 (HLCS), circ_000267 (SMG6), circ_006816 (ABHD17B), and circ_000005 (GRK3) were upregulated, whereas circ_022069 (ATXN2), circ_011916 (USP8), and circ_020661 (ABCA1) were downregulated in calves that received colostrum. Compared with the MK group, 76 circRNA were upregulated [e.g., circ_016046 (GOPC), circ_048271 (MICAL3), circ_006816 (ABHD17B), and circ_006817 $(C B L)]$ and 207 circRNA were downregulated [e.g., circ_037819 (SFT2D2), circ_017245 (COPG2), and circ_002883 $(R P T O R)]$ in the CO group (Supplemental Table S3; https://doi.org/10.3168/jds.2018-15942). Compared with the CT group, 147 circRNA were upregulated [including circ_012456 (HEPACAM2), circ_001283, and (RIC1) circ_044207 (RAB2B)] and 102 circRNA were downregulated [including circ_010492 (VPS4B), circ_010549 (IFRD1), and circ_010644 (CHST11)] in the MK group (Supplemental Table S4; https://doi .org/10.3168/jds.2018-15942).

To validate the differentially expressed circRNA obtained through the sequencing, 5 circRNA (circ_016046, circ_000267, circ_011322, circ_012456, and circ_033679) with different abundance and lengths were selected for quantification by reverse-transcrip- tion quantitative PCR (Table 1), demonstrating that circ_016046, circ_000267, circ_011322, and circ_033679 were upregulated in the $\mathrm{CO}$ group compared with the $\mathrm{CT}$ or MK groups, in agreement with the RNA sequencing data (Figure 2).

All of the differentially expressed circRNA identified among the 3 groups were further analyzed by hierarchical clustering analysis (Figure 3A) and principal component analysis (Figure 3B), demonstrating distinct expression patterns in the jejunum tissues according to the different feeding management of neonatal calves.

\section{Functional Analysis of Differentially Expressed CircRNA}

The most predominant GO terms related to the predicted target genes of differentially expressed circRNA in the jejunum tissue between the $\mathrm{CO}$ and $\mathrm{CT}$ groups were involved in response to cellular metabolic process, cellular biosynthetic process, regulation of cell differentiation, and response to extracellular stimulus in the biological process category; intracellular, organelle, cytoplasm, and macromolecular complex in the cellular component category; and catalytic activity, binding, and hydrolase activity in the molecular function category. Pathway analysis indicated that these differentially expressed circRNA were largely associated with metabolism-related pathways and cell cycle pathways (Supplemental Table S5; https://doi.org/10.3168/jds .2018-15942).

The predominant GO terms related to the differentially expressed circRNA between the $\mathrm{CO}$ and $\mathrm{MK}$ groups were related to macromolecule metabolic process, response to stress, intracellular signal transduction, vesicle-mediated transport, and growth according to biological processes; intracellular, cytoplasm, macromolecular complex, and organelle membrane in cellular components; and binding and enzyme activity in molecular functions. The significant pathways were assigned to the Rap1 signaling pathway, focal adhesion, cell cycle, ubiquitin-mediated proteolysis, and extracellular matrix-receptor interaction (Supplemental Table S6; https://doi.org/10.3168/jds.2018-15942).

Finally, the enriched GO categories for differentially expressed genes between the MK and CT groups were related to the biological regulation, tissue development, and cell morphogenesis in biological process terms; organelle, macromolecular complex, and cytoskeleton in cellular components; and binding and catalytic activity in molecular function. The pathways linked to these genes were assigned to ubiquitin-mediated proteolysis, cell cycle, and basal transcription factors (Supplemental Table S7; https://doi.org/10.3168/jds.2018-15942). 


\section{Regulation of CircRNA Acting as miRNA Sponges}

The circRNA harboring 1 or more miRNA-binding site were predicted to interact with 522 known and 229 novel miRNA. The circRNA-miRNA coexpression networks related to vesicle-mediated transport and endocytosis were constructed and analyzed using Cytoscape software, version 3.6.0 (http://cytoscape.org/), and
A CO vs. CT

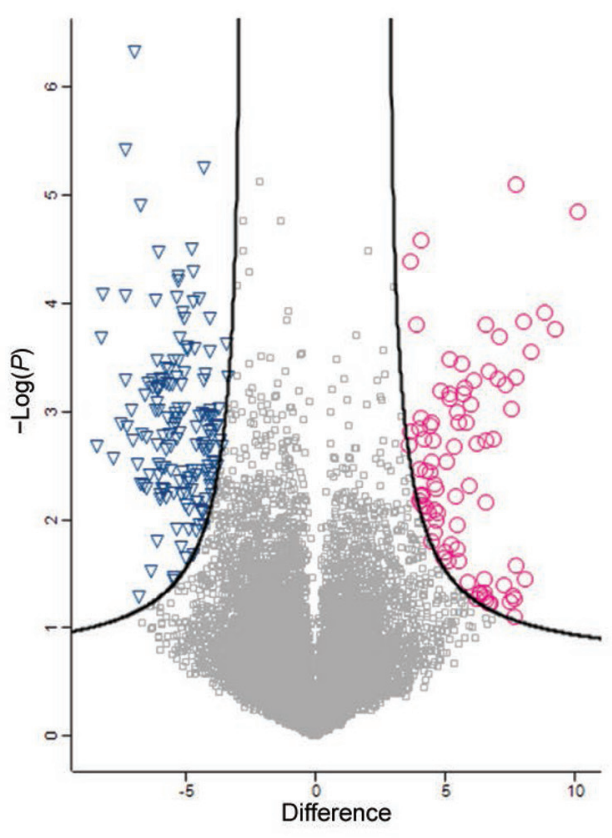

C MK vs. CT

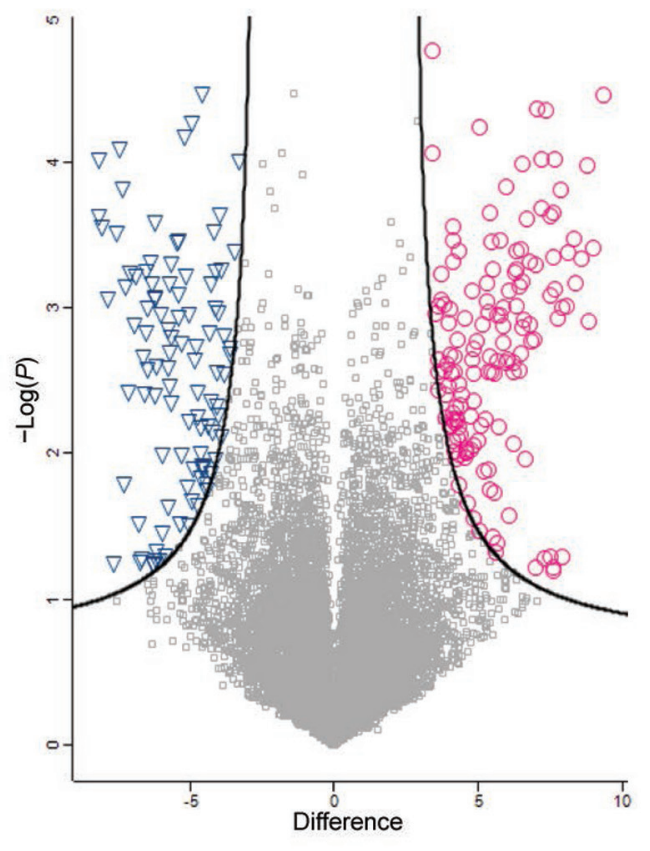

\section{B CO vs. MK}

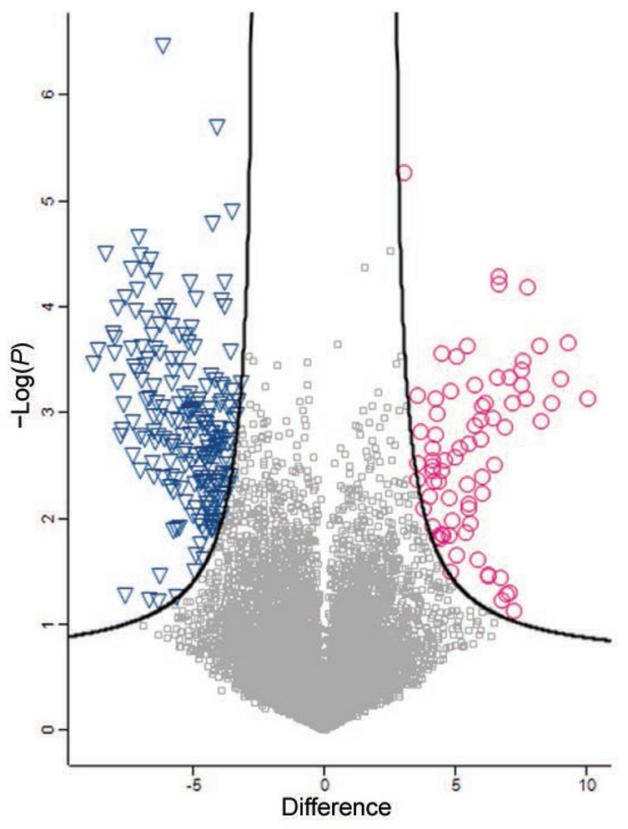

Figure 1. Volcano plots of differentially expressed circular RNA: CO versus CT (A), CO versus MK (B), and MK versus CT (C). Volcano

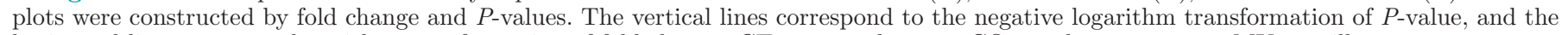
horizontal lines represent logarithm transformation of fold change. CT = control group; CO = colostrum group; MK = milk group. 

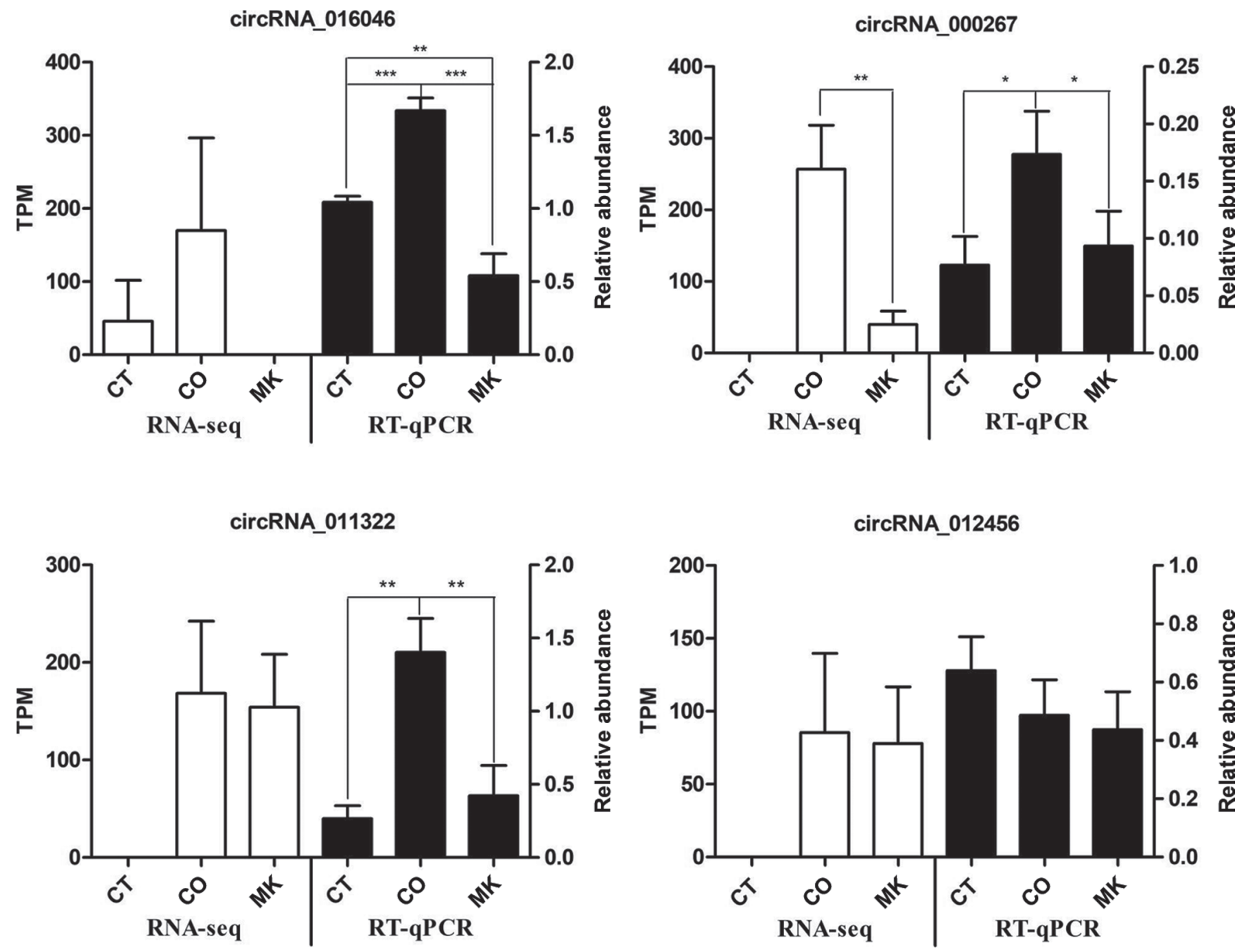

circRNA_033679

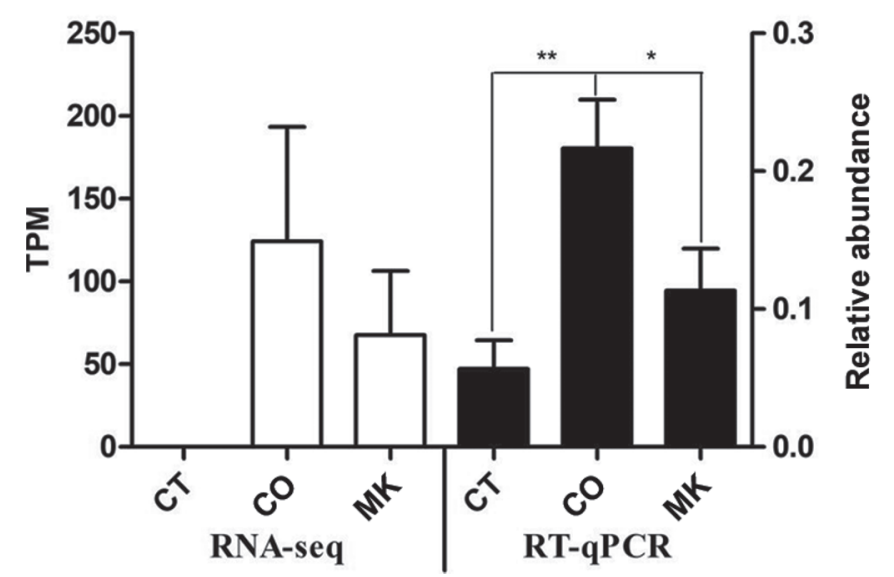

Figure 2. Reverse-transcription quantitative PCR (RT-qPCR) analysis of circular RNA (circRNA) expression. The expression of circRNA was defined based on the threshold cycle $(\mathrm{Ct})$, and the relative expression levels were calculated via the $2^{-\Delta \Delta \mathrm{Ct}}$ method. CT $=$ control group; $\mathrm{CO}=$ colostrum group; $\mathrm{MK}=$ milk group. RNA-seq $=\mathrm{RNA}$ sequencing; TPM $=$ transcript per million. Data are presented as the mean \pm SEM. ${ }^{*} P<0.05 ;{ }^{* *} P<0.01 ; * * * P<0.001$. 
A

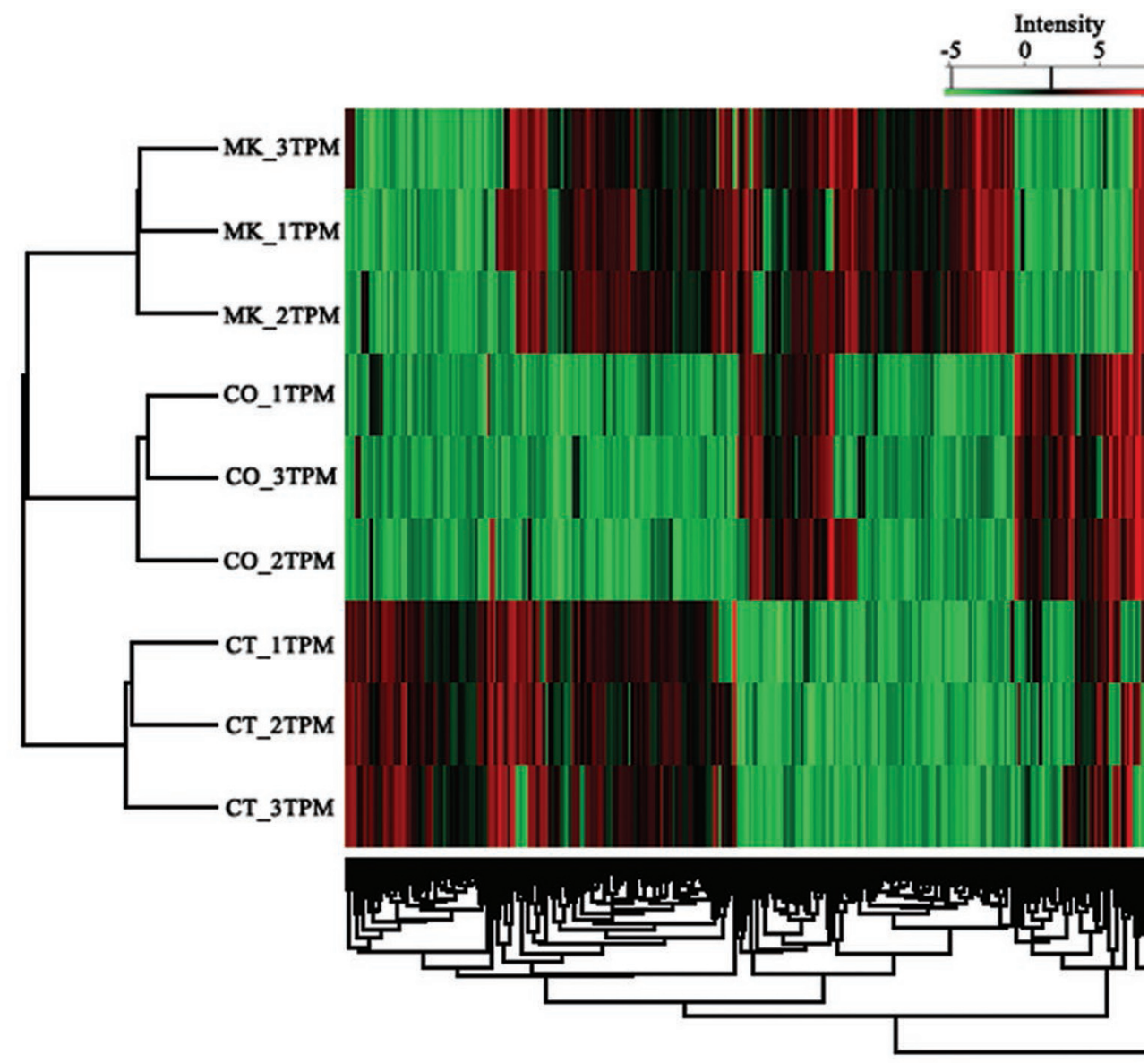

B

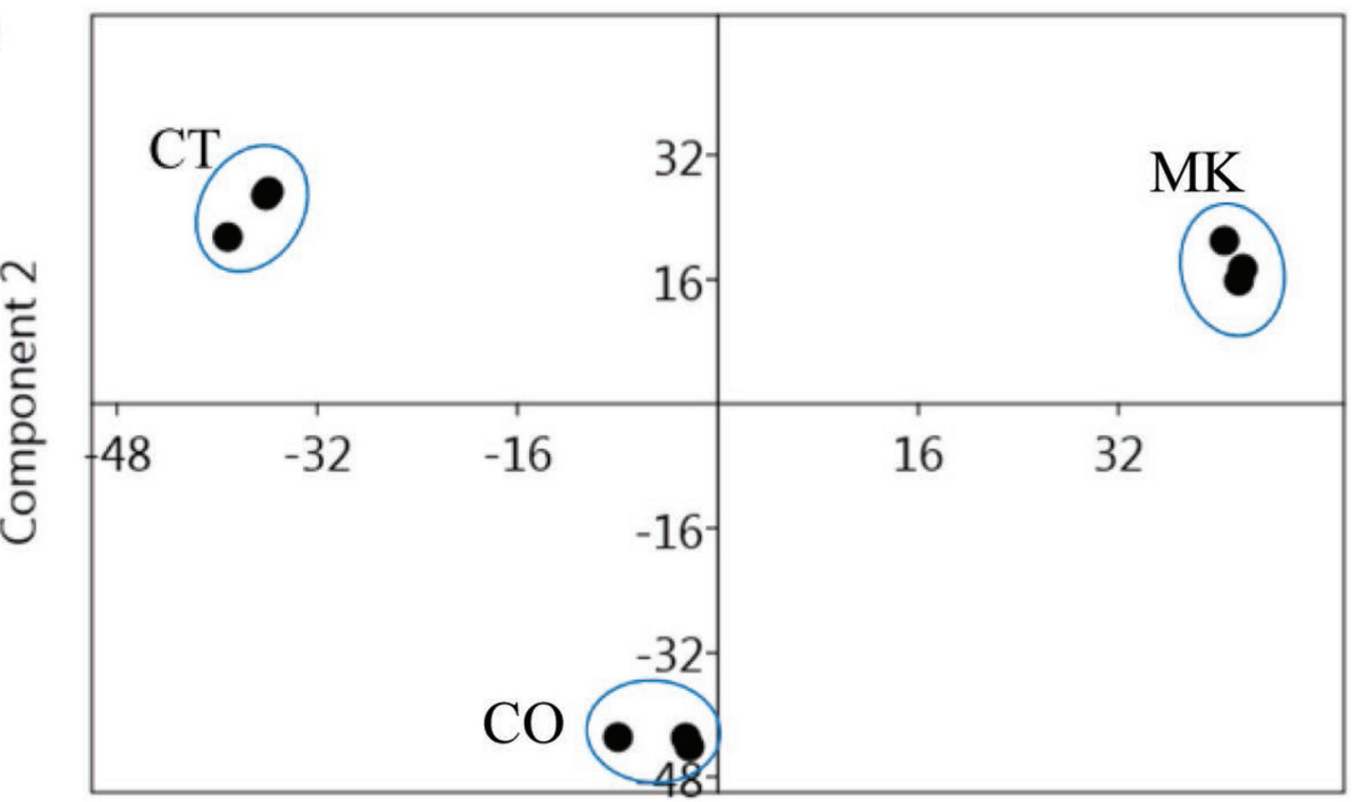

Component 1

Figure 3. Hierarchical clustering (A) and principal component analysis (B) of differentially expressed circular RNA (circRNA) of jejunum tissue in control calves (CT) or calves fed colostrum (CO) or milk (MK). TPM = transcript per million. 
118 miRNA interacted with 9 circRNA, including 4 upregulated and 5 downregulated circRNA in the network for the $\mathrm{CO}$ versus CT comparison. Similarly, in the network of the $\mathrm{CO}$ versus MK comparison, 162 miRNA interacted with 16 circRNA, including 5 upregulated and 11 downregulated circRNA (Supplemental Figure S3; https://doi.org/10.3168/jds.2018-15942). Further, the circRNA-miRNA-mRNA coexpression network was predicted, and the molecular interactions of circ_000781 (HIP1) with 4 miRNA (miR-152, miR-195, miR-370, and miR-412) and target genes are shown in Figure 4.

\section{DISCUSSION}

In the current study, the expression profile of circRNA in the small intestine of neonatal calves was first mapped using an RNA sequencing approach, and clear differences were identified in expression patterns according to nutrient source in early life (mature milk vs. colostrum). Recently, the circRNA profiles in the mammary gland of dairy cows (Zhang et al., 2016) and in the testes of neonatal and adult cattle (Gao et al., 2018) were investigated. However, little information is available concerning the expression profiles of circRNA in the small intestine of neonatal calves in response to colostrum intake. Our results may provide novel insight into the circRNA profiles of the neonatal intestine when colostrum passes through the gastrointestinal tract, thus enabling further investigation of the functions of circRNA.

The differentially expressed circRNA identified in the present study were shown to be involved in various

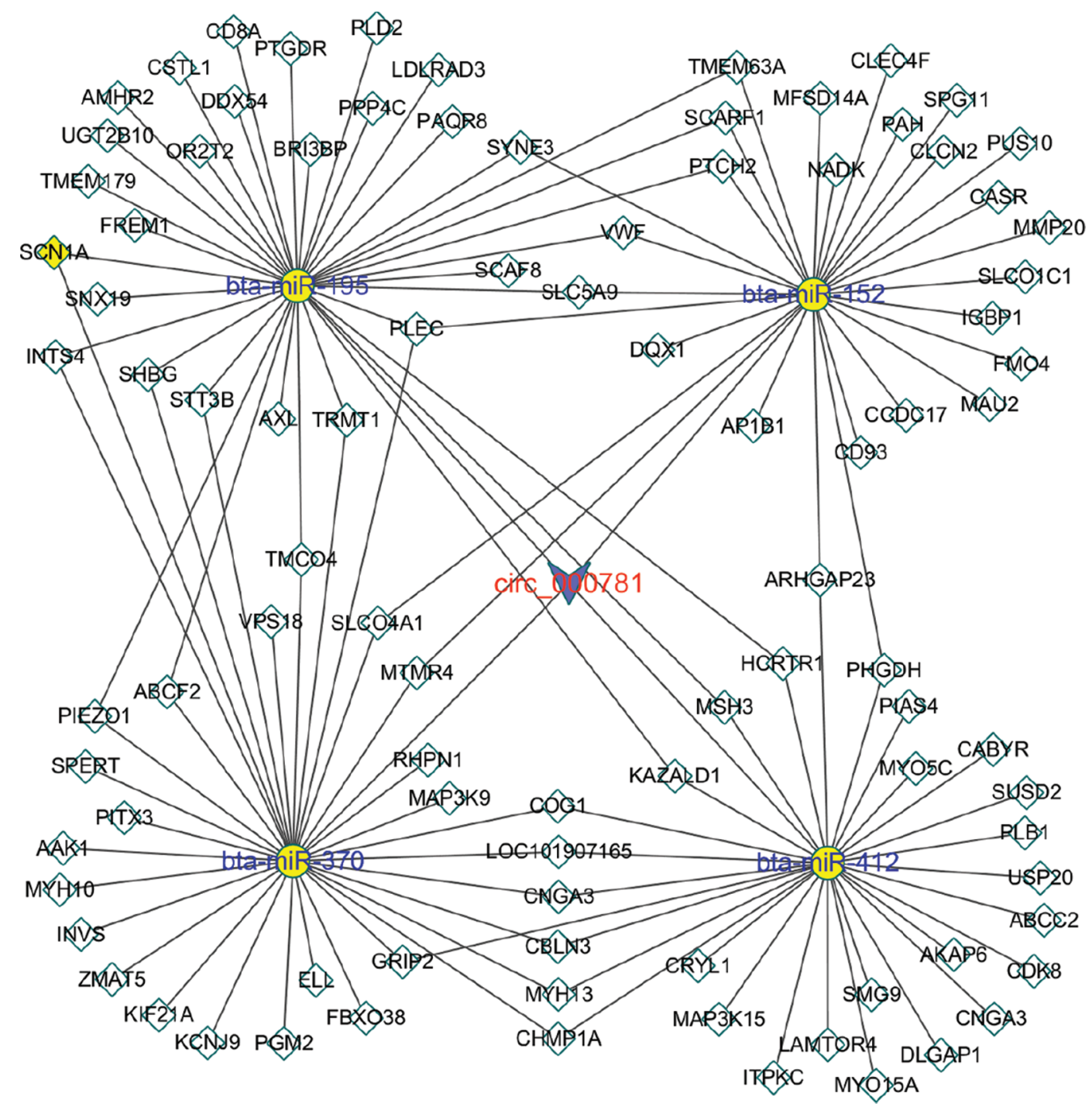

Figure 4. The circRNA-miRNA-mRNA coexpression network of predicted circ_000781 targets within the top 4 miRNA-mRNA. Triangle shows circular RNA (circRNA), circle shows microRNA (miRNA), and diamond shows mRNA. 
biological functions, according to bioinformatics analysis of their predicted parental genes. Th GO analysis indicated that most of the target genes of the circRNA influenced by colostrum feeding were associated with cellular metabolic process, response to stimulus, vesicle-mediated transport, and growth. Several colostrum components can be transported across the intestine into the blood in neonatal calves (Blum and Hammon, 2000; Yang et al., 2015), serving as a unique source of nutrients and bioactive components to promote development and health. In particular, because colostral IgG is crucial for the passive immune transfer in neonatal calves, the biological process of endocytosis or vesiclemediated transport in the small intestine is essential for transporting colostrum components. In line with the bioinformatics analysis, we found that the expression levels of several circRNA associated with endocytosis or vesicle trafficking, such as AHI1, GRK3, HIP1, and $G O P C$, increased in the jejunum tissue in calves that received colostrum.

Huntington interacting protein 1 (HIP1) is a component of the clathrin coat and has been considered to be a regulator of clathrin assembly via direct binding to the regulatory region of the clathrin gene while also playing a role in receptor-mediated endocytosis (Waelter et al., 2001; Legendre-Guillemin et al., 2005). Moreover, the presence of clathrin at the microvillous membrane of neonatal enterocytes was implicated in the receptormediated transport of IgG in calves (Jochims et al., 1994). G protein-coupled receptor 3 mRNA has also been detected in the rat gastrointestinal tract (Nedi et al., 2011), and the overexpression of GRK2, GRK3, and GRK 4 was found to enhance adrenomedullin-induced internalization of receptor activity-modifying proteins (Kuwasako et al., 2013). Abelson helper integration site 1 (AHI1) is a modular protein that contains $1 \mathrm{SH} 3$ motif and 7 WD40 repeats (Jiang et al., 2002) and has been suggested to be involved in vesicular trafficking via interaction with Rab8a. Moreover, defects in the trafficking of endocytic vesicles from the plasma membrane to the Golgi and back to the plasma membrane were observed in AHI1-knockdown cells (Hsiao et al., 2009), suggesting that AHI1 is indispensable in the vesicular trafficking process. Golgi-associated PDZ coiled-coil motif containing (GOPC) is a Frizzledinteracting protein and has been proposed to play a role in the vesicle transport from the Golgi apparatus to the plasma membrane (Yao et al., 2001; Cheng et al., 2002). This suggestion was further supported by 2 studies (Bock et al., 1997; Charest et al., 2001) that independently demonstrated an interaction of GOPC with syntan-6, which mediates a trans-Golgi network trafficking event. In addition, we found that the expres- sion level of ATXN2 was decreased when calves ingested colostrum. Ataxin-2 (ATXN2) is ubiquitously expressed, predominantly located in the Golgi apparatus, and is involved in multiple cellular pathways (Huynh et al., 2003). For example, endocytic internalization was shown to be inhibited via direct interaction of ATXN2 with SH3 motif-containing proteins, and ATXN2 deficiency significantly enhanced epidermal growth factor receptor endocytosis in embryonic fibroblasts (Nonis et al., 2008; Drost et al., 2013). Overall, this background suggests that the altered circRNA in the small intestine might contribute to improving the receptor-mediated transport of colostrum components.

The expression levels of circRNA were also associated with the cellular metabolic process and growth. For instance, the expression levels of HLCS, HIPK1, and ARHGAP18 increased in the jejunum tissue when neonates received colostrum. Holocarboxylase synthetase (HLCS) is an enzyme that catalyzes biotin incorporation into carboxylases and is involved in various cellular metabolic pathways, such as gluconeogenesis, fatty acid synthesis, and leucine catabolism (Wolf, 2001). Deficient HLCS activity may result in reduced activity of multiple carboxylases, leading to metabolic acidosis, hypotonia, and convulsions (Baumgartner and Suormala, 1997). Homeodomain-interacting protein kinase (HIPK) 1 is a Ser/Thr kinase that interacts with homeobox proteins and other transcription factors, acting as transcriptional coactivators or corepressors; HIPK (HIPK1, HIPK2, HIPK3, and HIPK4) contribute to regulate several cellular processes, such as proliferation, apoptosis, and development (Schmitz et al., 2014). Homeodomain-interacting protein kinase 1 was shown to phosphorylate and activate p53, resulting in the enhancement of p53-dependent transcription for cell growth regulation (Kondo et al., 2003). Rho GTPase-activating protein 18 (ARHGAP18) has many biological functions, including promotion of cell proliferation (Neisch et al., 2013) and control of cell shape and motility (Maeda et al., 2011). Knockdown and overexpression of ARHGAP18 resulted in the inhibition and promotion of cell migration, respectively (Maeda et al., 2011), indicating that ARHGAP18 may play an important role in regulating growth and development. Consistent with these results, intestinal development (e.g., villus length and width and mucosal thickness) was reported to be enhanced in neonatal calves that received colostrum (Blättler et al., 2001; Yang et al., 2015; Zhao and Yang, Anhui Academy of Agricultural Sciences, Hefei, China; unpublished data). However, the mechanism and roles of these circRNA and their target genes in endocytosis, metabolism, and growth of the neonatal intestine require further investigation. 
Circular RNA might also regulate the function of miRNA acting as competing endogenous RNA (Memczak et al., 2013; Kulcheski et al., 2016). For example, bovine casein-related circRNA (CSN1SI) was considered an endogenous miR-2284 family sponge for regulating casein translation (Zhang et al., 2016). In addition, the circRNA chi_circ_008219 was shown to serve as a sponge for 3 ovarian follicle-related miRNA (miR-483, miR-34c-5p, and miR-1468-3p) to regulate goat ovarian follicular development (Tao et al., 2017). We identified that a single circRNA could harbor various target sites for different miRNA and mRNA. For example, circ_000781 (HIP1) contained 25 miRNA potential binding sites, including those for miR-152, miR-195, miR-370, and miR-412, and was upregulated in the jejunum tissue when newborn calves ingested colostrum. Because HIP1 was suggested to be involved in receptor-mediated endocytosis (Waelter et al., 2001; Legendre-Guillemin et al., 2005), it seems likely that circRNA act as miRNA sponges to regulate gene expression for the transport of colostrum components, which in turn play a crucial role in the early life of neonates.

\section{CONCLUSIONS}

The circRNA profiles in the jejunum tissue of neonatal calves were mapped by RNA sequencing, demonstrating that circRNA expression is markedly affected by colostrum or milk feeding. In total, 36,829 circRNA were identified, 655 of which were differentially expressed according to feeding type. The GO and Kyoto Encyclopedia of Genes and Genomes biological pathway analysis indicated that the target genes of circRNA are mainly involved in macromolecule metabolism, response to stress, vesicle-mediated transport, and developmental growth. Several differentially expressed circRNA could also interact with miRNA to regulate target gene expression for colostrum macromolecule transport. These findings contribute to gaining a better understanding of the mechanism of digestion and absorption of colostrum components during the early life of calves, providing preliminary data for further studies on small intestine nutrition.

\section{ACKNOWLEDGMENTS}

The study was financially supported by the National Natural Science Foundation of China (No. 31572434; Beijing), the Special Fund for Agro-Scientific Research in the Public Interest (No. 201403071; Beijing), and Youth Innovation Fund of Anhui Academy of Agricultural Sciences (No. 16B0408; Hefei).

\section{REFERENCES}

Bartel, D. P. 2009. MicroRNAs: Target recognition and regulatory functions. Cell 136:215-233.

Batista, P. J., and H. Y. Chang. 2013. Long noncoding RNAs: Cellular address codes in development and disease. Cell 152:1298-1307.

Baumgartner, E. R., and T. Suormala. 1997. Multiple carboxylase deficiency: Inherited and acquired disorders of biotin metabolism. Int. J. Vitam. Nutr. Res. 67:377-384.

Blättler, U., H. M. Hammon, C. Morel, C. Philipona, A. Rauprich, V. Romé, I. Le Huërou-Luron, P. Guilloteau, and J. W. Blum. 2001. Feeding colostrum, its composition and feeding duration variably modify proliferation and morphology of the intestine and digestive enzyme activities of neonatal calves. J. Nutr. 131:1256-1263.

Blum, J. W., and H. Hammon. 2000. Colostrum effects on the gastrointestinal tract, and on nutritional, endocrine and metabolic parameters in neonatal calves. Livest. Prod. Sci. 66:151-159.

Bock, J. B., J. Klumperman, S. Davanger, and R. H. Scheller. 1997. Syntaxin 6 functions in trans-Golgi network vesicle trafficking. Mol. Biol. Cell 8:1261-1271.

Charest, A., K. Lane, K. Mcmahon, and D. E. Housman. 2001. Association of a novel PDZ domain-containing peripheral Golgi protein with the Q-SNARE (Q-soluble N-ethylmaleimide-sensitive fusion protein (NSF) attachment protein receptor) protein syntaxin 6. J. Biol. Chem. 276:29456-29465.

Cheng, J., B. D. Moyer, M. Milewski, J. Loffing, M. Ikeda, J. E. Mickle, G. R. Cutting, M. Li, B. A. Stanton, and W. B. Guggino. 2002. A Golgi-associated PDZ domain protein modulates cystic fibrosis transmembrane regulator plasma membrane expression. J. Biol. Chem. 277:3520-3529.

Conneely, M., D. P. Berry, J. P. Murphy, I. Lorenz, M. L. Doherty, and E. Kennedy. 2014. Effect of feeding colostrum at different volumes and subsequent number of transition milk feeds on the serum immunoglobulin $\mathrm{G}$ concentration and health status of dairy calves. J. Dairy Sci. 97:6991-7000.

Drost, J., D. Nonis, F. Eich, O. Leske, E. Damrath, E. R. Brunt, I. Lastres-Becker, R. Heumann, J. Nowock, and G. Auburger. 2013. Ataxin-2 modulates the levels of Grb2 and Src but not ras signaling. J. Mol. Neurosci. 51:68-81.

Gao, Y., M. Wu, Y. Fan, S. Li, Z. Lai, Y. Huang, X. Lan, C. Lei, H. Chen, and R. Dang. 2018. Identification and characterization of circular RNAs in Qinchuan cattle testis. R. Soc. Open Sci. $5: 180413$.

Georgiev, I. P. 2008. Differences in chemical composition between cow colostrum and milk. Bulg. J. Vet. Med. 11:3-12.

Godden, S. 2008. Colostrum management for dairy calves. Vet. Clin. North Am. Food Anim. Pract. 24:19-39.

Guttman, M., and J. L. Rinn. 2012. Modular regulatory principles of large non-coding RNAs. Nature 482:339-346.

Hsiao, Y. C., Z. J. Tong, J. E. Westfall, J. G. Ault, P. S. Page-McCaw, and R. J. Ferland. 2009. Ahi1, whose human ortholog is mutated in Joubert syndrome, is required for Rab8a localization, ciliogenesis and vesicle trafficking. Hum. Mol. Genet. 18:3926-3941.

Huynh, D. P., H. T. Yang, H. Vakharia, D. Nguyen, and S. M. Pulst. 2003. Expansion of the polyQ repeat in ataxin-2 alters its Golgi localization, disrupts the Golgi complex and causes cell death. Hum. Mol. Genet. 12:1485-1496.

Jain, A. K., I. J. Sharma, M. A. Quadri, R. K. Tripathi, R. G. Agrawal, and A. Mishra. 2007. Comparative feature of buffalo's and cow's colostrum vis-à-vis their sera samples. Indian J. Dairy Sci. 60:199-201.

Jeck, W. R., and N. E. Sharpless. 2014. Detecting and characterizing circular RNAs. Nat. Biotechnol. 32:453-461.

Jiang, X., Z. Hanna, M. Kaouass, L. Girard, and P. Jolicoeur. 2002 Ahi-1, a novel gene encoding a modular protein with WD40-repeat and SH3 domains, is targeted by the Ahi-1 and Mis-2 provirus integrations. J. Virol. 76:9046-9059.

Jochims, K., F. J. Kaup, W. Drommer, and M. Pickel. 1994. An immunoelectron microscopic investigation of colostral IgG absorption across the intestine of newborn calves. Res. Vet. Sci. 57:75-80. 
Kertz, A. F., T. M. Hill, J. D. Quigley, A. J. Heinrichs, J. G. Linn, and J. K. Drackley. 2017. A 100-year review: Calf nutrition and management. J. Dairy Sci. 100:10151-10172.

Kondo, S., Y. Lu, M. Debbas, A. W. Lin, I. Sarosi, A. Itie, A. Wakeham, J. Tuan, C. Saris, G. Elliott, W. Ma, S. Benchimol, S. W Lowe, T. W. Mak, and S. K. Thukral. 2003. Characterization of cells and gene-targeted mice deficient for the p53-binding kinase homeodomain-interacting protein kinase 1 (HIPK1). Proc. Natl. Acad. Sci. USA 100:5431-5436.

Kulcheski, F. R., A. P. Christoff, and R. Margis. 2016. Circular RNAs are miRNA sponges and can be used as a new class of biomarker. J. Biotechnol. 238:42-51.

Kuwasako, K., D. L. Hay, S. Nagata, M. Murakami, K. Kitamura, and J. Kato. 2013. Functions of third extracellular loop and helix 8 of Family B GPCRs complexed with RAMPs and characteristics of their receptor trafficking. Curr. Protein Pept. Sci. 14:416-428.

Legendre-Guillemin, V., M. Metzler, J. F. Lemaire, J. Philie, L. Gan, M. R. Hayden, and P. S. McPherson. 2005. Huntingtin interacting protein 1 (HIP1) regulates clathrin assembly through direct binding to the regulatory region of the clathrin light chain. J. Biol. Chem. 280:6101-6108.

Liang, G., N. Malmuthuge, T. B. McFadden, H. Bao, P. J. Griebel, P. Stothard, and L. L. Guan. 2014. Potential regulatory role of microRNAs in the development of bovine gastrointestinal tract during early life. PLoS One 9:e92592.

Maeda, M., H. Hasegawa, T. Hyodo, S. Ito, E. Asano, H. Yuang, K. Funasaka, K. Shimokata, Y. Hasegawa, M. Hamaguchi, and T. Senga. 2011. ARHGAP18, a GTPase-activating protein for RhoA, controls cell shape, spreading, and motility. Mol. Biol. Cell $22: 3840-3852$.

Memczak, S., M. Jens, A. Elefsinioti, F. Torti, J. Krueger, A. Rybak, L. Maier, S. D. Mackowiak, L. H. Gregersen, M. Munschauer, A. Loewer, U. Ziebold, M. Landthaler, C. Kocks, F. Le. Noble, and N. Rajewsky. 2013. Circular RNAs are a large class of animal RNAs with regulatory potency. Nature 495:333-338.

Moretti, D. B., W. M. Nordi, A. L. Lima, P. Pauletti, and R. Machado-Neto. 2013. Enterocyte IgG uptake in the small intestine of goat kids during the period of passive immunity acquisition. Small Rumin. Res. 114:182-187.

Nedi, T., P. J. White, I. M. Coupar, and H. R. Irving. 2011. Tissue dependent differences in G-protein coupled receptor kinases associated with 5-HT4 receptor desensitization in the rat gastrointestinal tract. Biochem. Pharmacol. 81:123-133.

Neisch, A. L., E. Formstecher, and R. G. Fehon. 2013. Conundrum, an ARHGAP18 orthologue, regulates RhoA and proliferation through interactions with Moesin. Mol. Biol. Cell 24:1420-1433.

Nonis, D., M. H. H. Schmidt, S. van de Loo, F. Eich, I. Dikic, J. Nowock, and G. Auburger. 2008. Ataxin-2 associates with the endocytosis complex and affects EGF receptor trafficking. Cell. Signal. 20:1725-1739.

Ontsouka, E. C., B. Korczak, H. M. Hammon, and J. W. Blum. 2004. Real-time PCR quantification of bovine lactase mRNA: Localization in the gastrointestinal tract of milk-fed calves. J. Dairy Sci. $87: 4230-4237$.

Osaka, I., Y. Matsui, and F. Terada. 2014. Effect of the mass of immunoglobulin (Ig)G intake and age at first colostrum feeding on serum IgG concentration in Holstein calves. J. Dairy Sci. 97:66086612

Qi, Y., X. Zhao, D. Huang, X. Pan, Y. Yang, H. Zhao, H. Hu, and G. Cheng. 2018. Exploration of the relationship between intestinal colostrum or milk, and serum metabolites in neonatal calves by metabolomics analysis. J. Agric. Food Chem. 66:7200-7208.

Qu, S., X. Yang, X. Li, J. Wang, Y. Gao, R. Shang, W. Sun, K. Dou, and H. Li. 2015. Circular RNA: A new star of noncoding RNAs. Cancer Lett. 365:141-148.

Robinson, J. T., H. Thorvaldsdottir, W. Winckler, M. Guttman, E. S. Lander, and G. Getz. 2011. Integrative genomics viewer. Nat. Biotechnol. 29:24-26.

Schmitz, M. L., A. Rodriguez-Gil, and J. Hornung. 2014. Integration of stress signals by homeodomain interacting protein kinases. Biol. Chem. 395:375-386.

Sharbati, S., M. R. Friedländer, J. Sharbati, L. Hoeke, W. Chen, A. Keller, P. F. Stähler, N. Rajewsky, and R. Einspanier. 2010. Deciphering the porcine intestinal microRNA transcriptome. BMC Genomics 11:275

Tao, H., Q. Xiong, F. Zhang, N. Zhang, Y. Liu, X. J. Suo, X. F. Li, Q. P. Yang, and M. X. Chen. 2017. Circular RNA profiling reveals chi_circ_0008219 function as microRNA sponges in pre-ovulatory ovarian follicles of goats (Capra hircus). Genomics 110:257-267.

Taulli, R., C. Loretelli, and P. P. Pandolfi. 2013. From pseudo-ceRNAs to circ-ceRNAs: A tale of cross-talk and competition. Nat. Struct. Mol. Biol. 20:541-543.

Trapnell, C., B. A. Williams, G. Pertea, A. Mortazavi, G. Kwan, and M. J. van Baren. 2010. Transcript assembly and quantification by RNA-Seq reveals unannotated transcripts and isoform switching during cell differentiation. Nat. Biotechnol. 28:511-515.

Waelter, S., E. Scherzinger, R. Hasenbank, E. Nordhoff, R. Lurz, H Goehler, C. Gauss, K. Sathasivam, G. P. Bates, H. Lehrach, and E. E. Wanker. 2001. The huntingtin interacting protein HIP1 is a clathrin and alpha-adaptin-binding protein involved in receptormediated endocytosis. Hum. Mol. Genet. 10:1807-1817.

Wolf, B. 2001. Disorders of biotin metabolism. Pages 3935-3962 in The Metabolic and Molecular Basis of Inherited Disease. C. R Scriver, A. L. Beaudet, W. S. Sly, and D. Valle, ed. McGraw-Hill, New York, NY.

Yang, M., Y. Zou, Z. H. Wu, S. L. Li, and Z. J. Cao. 2015. Colostrum quality affects immune system establishment and intestinal development of neonatal calves. J. Dairy Sci. 98:7153-7163.

Yao, R., T. Maeda, S. Takada, and T. Noda. 2001. Identification of a PDZ domain containing Golgi protein, GOPC, as an interaction partner of frizzled. Biochem. Biophys. Res. Commun. 286:771-778.

Zhang, C., H. Wu, Y. H. Wang, S. Q. Zhu, J. Q. Liu, X. T. Fang, and H. Chen. 2016. Circular RNA of cattle casein genes are highly expressed in bovine mammary gland. J. Dairy Sci. 99:4750-4760.

Zhang, Y., X. Q. Zhang, T. Chen, J. F. Xiang, Q. F. Yin, X. H. Xing, S. S. Zhu, L. Yang, and L. L. Chen. 2013. Circular intronic long noncoding RNAs. Mol. Cell 51:792-806.

Zhao, X. W., Y. X. Qi, D. W. Huang, X. C. Pan, G. L. Cheng, H. L. Zhao, and Y. X. Yang. 2018. Changes in serum metabolites in response to ingested colostrum and milk in neonatal calves, measured by nuclear magnetic resonance-based metabolomics analysis. J. Dairy Sci. 101:7168-7181. 